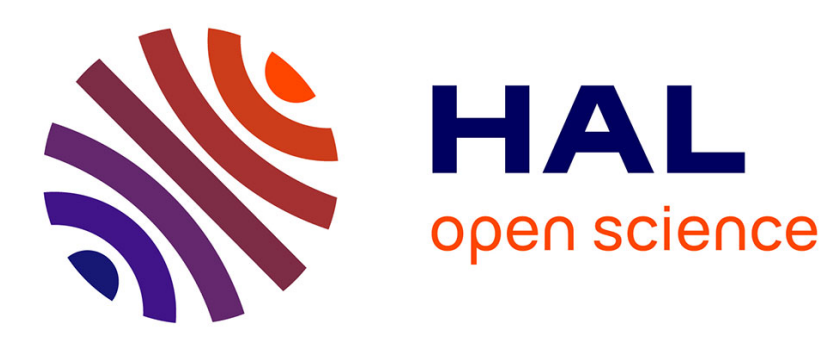

\title{
Polymer adsorption on surfactant monolayers and heterogeneous solid surfaces
}

David Andelman, Jean-François Joanny

\section{To cite this version:}

David Andelman, Jean-François Joanny. Polymer adsorption on surfactant monolayers and heterogeneous solid surfaces. Journal de Physique II, 1993, 3 (1), pp.121-138. 10.1051/jp2:1993115 . jpa00247805

\section{HAL Id: jpa-00247805 https://hal.science/jpa-00247805}

Submitted on 1 Jan 1993

HAL is a multi-disciplinary open access archive for the deposit and dissemination of scientific research documents, whether they are published or not. The documents may come from teaching and research institutions in France or abroad, or from public or private research centers.
L'archive ouverte pluridisciplinaire HAL, est destinée au dépôt et à la diffusion de documents scientifiques de niveau recherche, publiés ou non, émanant des établissements d'enseignement et de recherche français ou étrangers, des laboratoires publics ou privés. 
Classification

Physics Abstracts

$68.45-61.25-82.65$

\title{
Polymer adsorption on surfactant monolayers and heterogeneous solid surfaces
}

\author{
David Andelman ( ${ }^{1}$ ) and Jean-François Joanny $\left({ }^{2}\right)$ \\ ( ${ }^{1}$ ) School of Physics and Astronomy, Raymond and Beverly Sackler Faculty of Exact Sciences, \\ Tel-Aviv University, Ramat-Aviv 69978, Tel-Aviv, Israel \\ $\left({ }^{2}\right)$ Institut Charles Sadron, 6 rue Boussingault, 67083 Strasbourg Cedex, France \\ (Received 29 July 1992, accepted 6 October 1992)
}

\begin{abstract}
We study polymer adsorption on a flat but heterogeneous surface, in both cases where the heterogeneity is quenched or annealed. We always find that the heterogeneity of the adsorbing surface enhances adsorption. As an example of surface with annealed heterogeneity we consider either soluble surfactant monolayers (at a fixed chemical potential) or insoluble (at a fixed concentration) for attractive interactions between polymer and surfactant. Even if the monolayer is on average neutral for polymer adsorption an isolated polymer chain adsorbs via a local increase of the surfactant surface concentration. The adsorption of a polymer solution can induce phase transitions in an insoluble monolayer that phase separates into dense regions where the polymer adsorbs and dilute regions from which the polymer is depleted. Phase transitions induced by polymer adsorption can also occur for soluble monolayer. For a surface with periodic quenched heterogeneities we calculate the amount of adsorbed polymer as a function of the wavelength of the heterogeneity.
\end{abstract}

\section{Introduction.}

Polymer adsorption from solution plays a major role in a number of industrial problems such as colloidal stabilization, adhesion and lubrication, and has been the subject of numerous experimental and theoretical studies. Over the last few years a rather detailed description of the structure and properties of adsorbed polymer layers on "ideal" surfaces (in the sense of perfectly flat and chemically homogeneous) has been developed [1-5].

Since the adsorption of polymers is a cooperative effect, the polymers which are attracted by the surface are adsorbed strongly and form a thick adsorption layer. If the adsorption takes place from a semi-dilute polymer solution, the thickness of this layer is of the order of the correlation length $\xi$ of the bulk polymer solution. On the other hand, if the adsorption proceeds from a dilute solution, the thickness is of the order of the radius of gyration of an isolated chain and can, thus, be as large as a few hundred angströms. In the adsorbed layer, the polymer chains form loops with a broad distribution of sizes and the structure of the 
layer is self-similar; the monomer concentration profile decays as an inverse power law of the distance from the surface. This self-similar structure [3] accounts well for many of the observed properties of adsorbed polymer layers [2, 5].

Most real surfaces are, however, neither homogeneous in composition nor smooth. Although the heterogeneity may strongly influence the adsorption of polymer solutions, these non-ideal situations have received relatively little theoretical attention [6-12]. The aim of this paper is to study the effect of chemical heterogeneities on polymer adsorption while keeping the adsorbing surface flat.

Two types of adsorbing surfaces are considered: quenched and annealed heterogeneous surfaces [12]. A heterogeneous surface is quenched if the disorder characterizing the adsorbing surface is a "frozen" degree of freedom in the thermodynamic sense. A typical example is a solid surface with irreversibly attached chemical impurities which cannot laterally diffuse on the surface. A quenched disordered surface represents a different local environment for the adsorbing monomers. We model it by a surface interaction which has local fluctuations about a mean value. For random quenched disorder [13], one must first calculate the properties of the adsorbed polymer layer for a given realization of the surface interaction and then average these properties with respect to the disorder.

On the other hand, if the heterogeneity is annealed, the disorder of the adsorbing surface is at thermodynamic equilibrium. This is, for instance, the case of chemical heterogeneities that can freely diffuse laterally on a solid surface. Another example which we consider in details is a surfactant monolayer at an air-water interface. The annealed heterogeneity is then the surfactant concentration per unit area. The important issue to be addressed is how the surfactant-polymer coupling affects the polymer adsorption as well as the surfactant surface properties such as its plase transitions and surface tension.

Our paper is organized as follows. In the next section, we present general considerations on the thermodynamics of mixed polymer-surfactant systems. Then, in section 3 , we study the adsorption of a single polymer chain on a surfactant monolayer. Although this is a rather academic problem, it gives some physical insight into the problem of surfactant-polymer interactions. Section 4 is devoted to the adsorption of polymers from semi-dilute solutions on annealed heterogeneous surfaces, and more specifically on surfactant monolayers. The description of the polymer solution properties remains at a mean field level along the lines of the Edwards density functional approach of polymer adsorption [14, 15]. Section 5 considers periodic and quenched heterogeneous surfaces both in the case where the surface is neutral on average for the polymer adsorption and in the case where it is strongly adsorbing. The last section presents our conclusions and gives possible directions for future investigations as well as experimental implications.

\section{Thermodynamics of surfactant-polymer systems.}

2.1 FreE ENERGy. - Mixtures of polymers and surfactants show a rich variety of phenomena in bulk solutions [16]. Polymers are, for instance, added in some applications as defoaming agents in surfactant solutions. Here, we concentrate, though, on a relatively simpler situation [17-19] where the interaction between polymer and surfactant takes place at a flat interface (e.g. air-water or oil-water interface). The surfactant forms either a soluble or an insoluble monolayer [20] whereas the polymer is soluble in the solution but adsorbs on the surface. The phenomenological free energy of the mixture $F_{\mathrm{t}}=F_{\mathrm{p}}+F_{\mathrm{s}}+F_{\mathrm{ps}}$ is then a sum of a polymer contribution, a surfactant contribution and a coupling term, respectively.

For an insoluble Langmuir monolayer, the total number of surfactant molecules is fixed and their concentration $c_{s}$ is a conserved order parameter. The other situation is a soluble Gibbs 
monolayer in coexistence with a bulk surfactant reservoir. Here, the surfactant concentration $c_{\mathbf{s}}$ is a non-conserved order parameter and is obtained by minimization of the free energy $F_{\mathrm{s}}$ at constant chemical potential imposed by the bulk solution.

The polymer-surfactant interaction $F_{\mathrm{ps}}$ depends both on the local surfactant concentration $c_{\mathrm{s}}$ and the polymer concentration (per unit volume) $c_{\mathrm{p}}$ at the surface. The most simple type of interaction is a bilinear coupling (per unit area and in units of $k_{\mathrm{B}} T$ )

$$
F_{\mathrm{ps}}=-a^{2}\left(\alpha c_{\mathrm{s}}+\gamma_{0}\right) c_{\mathrm{p}}
$$

where $\gamma_{0}$ describes the bare surface-polymer interaction, $\alpha$ is the direct polymer-surfactant interaction parameter and $a$ is the monomer size. Positive (negative) values of $\alpha$ indicate an attractive (repulsive) polymer-surfactant interaction. The coefficient $\alpha$ has units of length and when the molecular interaction is of the order of $k_{\mathrm{B}} T, \alpha$ is comparable to the monomer size $a$. In the following we consider only attractive interactions between surfactants and polymers, $\alpha>0$.

A special surface condition for the polymer occurs when $\alpha c_{s}^{*}+\gamma_{0}=0$. At this surfactant concentration $c_{\mathrm{s}}^{*}$, the polymer undergoes a surface phase transition (the "special transition"), and the surface is a neutral surface for polymer adsorption. For $\alpha>0$, the surface changesover at the concentration $c_{s}^{*}$ from depletion at lower surfactant concentration to adsorption at higher values. In general, the coefficients $\alpha$ and $\gamma_{0}$ can be functions of temperature and $c_{\mathrm{s}}$. In this work we consider them as constants; hence, the neutral surface condition $c_{\mathbf{s}}^{*}=-\gamma_{0} / \alpha$ defines a vertical line in the temperature - surfactant concentration plane. We now discuss separately the thermodynamics of one single chain adsorbing on the monolayer and the case where many polymer chains form a continuous adsorption layer.

For single chain adsorption, the adsorbed polymer can change locally the surfactant concentration $c_{\mathrm{s}}$ from the overall imposed value $c_{0}$ (far away from the adsorbed chain) to a higher value (when $\alpha>0$ ) $c_{\mathrm{s}}=c_{0}+\delta c$. The imposed value $c_{0}$ does not depend on the polymer adsorption but only on the properties of the monolyer in the absence of polymer. As long as the monolayer is homogeneous (single phase), $F_{\mathrm{s}}\left(c_{\mathrm{s}}\right)$ is a convex function around $c_{\mathrm{s}}=c_{0}$ and the adsorbed state is determined by minimizing the grand canonical free energy $F_{\mathrm{t}}-\mu c_{\mathrm{s}}$ where the chemical potential $\mu$ is required to be equal in the adsorbed state and in the reference state: $\mu=\partial F_{\mathrm{s}} / \partial c_{0}=\partial F_{\mathrm{t}} / \partial c_{\mathrm{s}}$. If $F_{\mathrm{t}}\left(c_{\mathrm{s}}\right)>F_{\mathrm{s}}\left(c_{0}\right)$, the adsorbed state is metastable, the polymer chain does not adsorb and the monolayer remains in its reference state $c_{\mathrm{s}}=c_{0}$. If $F_{\mathrm{t}}\left(c_{\mathrm{s}}\right)<F_{\mathrm{s}}\left(c_{0}\right)$, the polymer adsorbs and locally the surfactant concentration is higher than $c_{0}$. Note that even for a neutral surface, $c_{0}=c_{s}^{*}$ adsorption is possible since the surface is no longer homogeneous. In cases where the pure surfactant monolayer separates into a dilute and a dense phase, the surfactant free energy $F_{s}\left(c_{0}\right)$ is not convex. For attractive polymer-surfactant interactions $(\alpha>0)$, the reference surfactant concentration is then that of the equilibrium dense phase and not $c_{0}$.

We turn now to the adsorption from a semi-dilute polymer solution [17]. The minimization of the total free energy $F_{\mathrm{t}}=F_{\mathrm{s}}+F_{\mathrm{p}}+F_{\mathrm{ps}}$ with respect to the polymer concentration profile yields an effective free energy that depends only on the surfactant concentration. The polymer adsorption is coupled to the surfactant phase transitions. For a soluble monolayer, the equilibrium surfactant concentration in the monolayer is given by further minimizing the free energy $F_{\mathrm{t}}$ with respect to $c_{\mathrm{s}}$. (It is larger than the reference surfactant concentration $c_{0}$ in the absence of polymer.) Polymer adsorption can induce a first-order transition by changing the relative stability of two local minima of the free energy $F_{\mathrm{t}}$.

For an insoluble monolayer, the concentrations of the two phases in equilibrium are given by the standard common tangent construction on the total free energy $F_{\mathrm{t}}$. The polymer 
adsorption can induce a phase transition between dilute surfactant regions from which the polymer is repelled and dense surfactant regions where the polymer is adsorbed.

Rather than calculating global phase diagrams, we often only investigate the stability of the homogeneous monolayer. This defines the spinodal line that lies inside the coexistence region; on this line the curvature of the free energy vanishes. For both soluble and insoluble monolayers it is given by

$$
\frac{\partial^{2} F_{\mathrm{t}}}{\partial c_{\mathrm{s}}^{2}}=F_{\mathrm{s}}^{\prime \prime}+F_{\mathrm{p}}^{\prime \prime}+F_{\mathrm{ps}}^{\prime \prime}=0
$$

Finally, we mention a specific form that can be used as the surfactant free energy $F_{\mathrm{s}}$. Within a Bragg-Williams theory (mean-field), the free energy of a surfactant monolayer is a sum of the enthalpy and entropy of mixing. Disregarding linear terms, $F_{\mathrm{s}}$ is written as

$$
F_{\mathrm{s}}=\varepsilon c_{\mathrm{s}}\left(1-c_{\mathrm{s}} A_{0}\right)+c_{\mathrm{s}} \log c_{\mathrm{s}}+\frac{1}{A_{0}}\left(1-c_{\mathrm{s}} A_{0}\right) \log \left(1-c_{\mathrm{s}} A_{0}\right)
$$

where $\varepsilon$ is the interaction parameter of the surfactant on the surface and $A_{0}$ is the closepacking area. Phase diagrams can be obtained by studying $F_{\mathrm{s}}$ directly. Alternatively, $F_{\mathrm{s}}$ can be expressed as a Landau expansion in powers of $\delta c=c_{s}-c_{0}$ around a reference surfactant concentration $c_{0}$. The origin of the surfactant chemical potential can be chosen in order to eliminate the linear term in the expansion

$$
F_{\mathrm{s}}=F_{0}\left(c_{0}\right)+\frac{1}{2} t \delta c^{2}+\frac{1}{3} \lambda_{3} \delta c^{3}+\frac{1}{4} \lambda_{4} \delta c^{4} .
$$

In the Bragg-Williams approximation,

$$
\begin{aligned}
t & =-2 \varepsilon A_{0}+\frac{1}{c_{0}}+\frac{1}{A_{0}^{-1}-c_{0}} \\
\lambda_{3} & =-\frac{1}{2 c_{0}^{2}}+\frac{1}{2\left(A_{0}^{-1}-c_{0}\right)^{2}} \\
\lambda_{4} & =\frac{1}{3 c_{0}^{3}}+\frac{1}{3\left(A_{0}^{-1}-c_{0}\right)^{3}}
\end{aligned}
$$

In the following we choose for simplicity the concentration $c_{0}$ of the reference state to be the critical concentration $c_{k}=1 /\left(2 A_{0}\right)$. Then, the expansion has only even powers of $\delta c$ : $t=2 A_{0}(2-\varepsilon), \lambda_{3}=0$ and $\lambda_{4}=16 A_{0}^{3} / 3$. Since the Landau expansion is more general than the Bragg-Williams theory, we use the general coefficients $t, \lambda_{4}$ throughout the paper. In Appendix $A$ we also give an example of single chain adsorption when the surfactant reference concentration $c_{0}$ is off-critical.

2.2 SURfaCE TENSION. - One of the most easily measurable properties of surfactant and polymer systems is their surface tension, which can be measured as function of surfactant and polymer concentrations and temperature. For an insoluble Langmuir monolayer, changes in the surface tension can be measured in a Langmuir trough as function of the area per molecule on the surface.

The total surface tension $\sigma$ has several contributions, the first being the bare water/air surface tension $\sigma_{0}$. For an insoluble Langmuir monolayer, the surfactant and polymer contributions to the surface tension, $\sigma-\sigma_{0}$, are derived from the total interfacial free energy $F_{\mathrm{t}}$, keeping in mind that the surfactant concentration is fixed whereas the polymer is kept at a constant chemical potential

$$
\sigma-\sigma_{0}=F_{\mathrm{t}}-c_{\mathrm{s}} \frac{\partial F_{\mathrm{t}}}{\partial c_{\mathrm{s}}}
$$


Using the interaction energy $F_{\mathrm{ps}}$ of equation (2.1), $\sigma-\sigma_{0}$ can be rewritten as

$$
\sigma-\sigma_{0}=\sigma_{\mathrm{s}}+\sigma_{\mathrm{p}}+a^{2} \alpha c_{\mathrm{s}} c_{\mathrm{p}}=\sigma_{\mathrm{s}}+F_{\mathrm{p}}-a^{2} \gamma_{0} c_{\mathrm{p}}
$$

where $\sigma_{\mathrm{s}}=F_{\mathrm{s}}-c_{\mathrm{s}} \partial F_{\mathrm{s}} / \partial c_{\mathrm{s}}$ is the (negative) contribution of the pure surfactant monolayer, and $\sigma_{\mathrm{p}}=F_{\mathrm{p}}+F_{\mathrm{ps}}$ is the contribution of a polymer solution adsorbing on a surface with a given adsorption interaction parameter $\gamma=\alpha c_{s}+\gamma_{0}$. Notice, as is seen from equation (2.7), that the surface tension of the monolayer $\sigma-\sigma_{0}$ is always larger than the sum of the contributions of the polymer and the surfactant $\sigma_{\mathrm{s}}+\sigma_{\mathrm{p}}$. In certain cases $a^{2} \alpha c_{\mathrm{s}} c_{\mathrm{p}}$ is larger than the surface tension of the pure surfactant monolayer $\sigma_{s}$, even though the polymer-surfactant interaction is attractive. This surprising result is due to the fact that, as shown below, the adsorbed polymer induces effective attractive interactions between the surfactant molecules, reducing the surfactant surface pressure and increasing the surface tension. The polymer surface excess $\Gamma$ is related to the surface tension by Gibbs law.

$$
\left.\frac{\partial \sigma}{\partial \mu_{\mathrm{p}}}\right|_{\mu_{\mathrm{s}}}=-\Gamma
$$

where $\mu_{\mathrm{p}}$ is the polymer bulk chemical potential and the derivative is taken at constant chemical potential of the surfactant in the monolayer. For insoluble monolayers, it is usually the surfactant concentration $c_{s}$ and not the chemical potential which is held constant; equation (2.8) can then be recasted as

$$
\left.\frac{\partial \sigma}{\partial \mu_{\mathrm{p}}}\right|_{c_{\mathrm{s}}}=-\Gamma+a^{2} \alpha c_{\mathrm{s}} \frac{\partial c_{\mathrm{p}}}{\partial \mu_{\mathrm{p}}}
$$

The last term on the right hand side is usually not accessible experimentally and the measurement of the surface tension does not allow a direct measurement of the polymer surface excess. Note also that this term is positive and can be larger than the polymer surface excess; the surface tension decreases then with the polymer chemical potential or equivalently with the polymer bulk concentration $c_{\mathrm{b}}$.

For a soluble Gibbs monolayer the free energies that we have considered are grand canonical free energies at fixed chemical potential for both the polymer and the surfactant. The contribution of the monolayer to the surface tension is then its free energy per unit area

$$
\sigma-\sigma_{0}=F_{\mathrm{p}}+F_{\mathrm{s}}+F_{\mathrm{ps}}
$$

Since the equilibrium concentration in the monolayer is larger than the value in the absence of polymer, the surface tension $\sigma-\sigma_{0}$ is larger than the sum of the pure surfactant and pure polymer contributions. For a soluble monolayer, the chemical potential of the surfactant is held constant and the polymer surface excess is accessible from Gibbs law, equation (2.8).

\section{Single chain adsorption on a surfactant monolayer.}

Consider the adsorption of one single polymer chain on a surfactant monolayer with an average concentration $c_{0}$ at the air-water interface. In actual experiments, even when the adsorbing polymer solution has a concentration much smaller than the overlap concentration, the adsorbed chains overlap and form a continuous surface layer. Single chain adsorption can be observed only for unrealistically low concentrations or for short enough polymer chains but give some insight on the role of heterogeneities on polymer adsorption.

For simplicity, we consider a Gaussian polymer chain of $N$ monomers of size $a$, while neglecting excluded volume interactions. A more complete treatment using scaling arguments 
accounting for excluded volume effects has been carried out but gives essentially the same qualitative results. In order to examine in detail the role of surface disorder, we first consider a neutralsurface for polymer adsorption and then the general case (either attractive or repulsive surface).

3.1 The neutral surface. - We first treat a Langmuir monolayer whose overall concentration $c_{0}$ corresponds exactly to that of a neutral surface. Recalling that $c_{0}$ is taken as the surfactant concentration at its critical value, $c_{0}=c_{k}$, we can still tune the parameters $\gamma_{0}$ and $\alpha$ of equation (2.1) so that the "special transition" line $c_{\mathrm{s}}^{*}=\gamma_{0} / \alpha$ coincides exactly with $c_{0}$. In this case the affinity of the interface for the polymer and the solvent is the same. If the surfactant concentration remains homogeneous, the polymer does not adsorb and its concentration profile is constant. However, the polymer chain is coupled to concentration fluctuations in the monolayer and can adsorb by changing locally the surfactant concentration. As the average monolayer is neutral, the coupling term $F_{\mathrm{ps}}$ between the polymer and the surfactant is proportional to $\delta c$ and to the local monomer concentration $1 /\left(a^{2} D\right)$ in contact with the surface, equation (2.1)

$$
F_{\mathrm{ps}}=-\frac{\alpha \delta c}{D}
$$

As explained above, the total free energy is the sum of two additional contributions: $F_{\mathrm{t}}=$ $F_{\mathrm{p}}+F_{\mathrm{s}}+F_{\mathrm{ps}}$. For a single Gaussian chain confined within a layer of thickness $D$ from the surface, the confinement free energy $F_{\mathrm{p}}$ (per unit area and in units of $k_{\mathrm{B}} T$ ) is

$$
F_{\mathrm{p}}=\frac{1}{2 D^{2}}
$$

since the area occupied by the Gaussian chain on the surface is $N a^{2}$. The surfactant free energy $F_{\mathrm{s}}$, equation (2.4), expanded around the critical concentration $c_{\mathrm{k}}$ in powers of $\delta c=c_{\mathrm{s}}-c_{\mathrm{k}}=$ $c_{\mathbf{s}}-c_{0}$ reads

$$
F_{\mathrm{s}}=\frac{1}{2} t \delta c^{2}+\frac{1}{4} \lambda_{4} \delta c^{4}
$$

Minimizing the total free energy functional with respect to the layer thickness $D$, the optimum thickness $D^{*}$ is inversely proportional to $\delta c, D^{*}=1 /(\alpha \delta c)$. The total free energy can now be written as a function of $\delta c$

$$
F_{\mathrm{t}}=\frac{1}{2} \delta c^{2}\left(t-\alpha^{2}\right)+\frac{1}{4} \lambda_{4} \delta c^{4}
$$

The monolayer shows thus a continuous transition for a critical value of $t, t_{\mathrm{c}} \equiv \alpha^{2}$. If $t>\alpha^{2}$, the minimum of the free energy is obtained for a homogeneous surface concentration, $\delta c=0$, and there is no adsorption since the average surface is assumed to be neutral. If $t<\alpha^{2}$, the minimum of $F_{\mathrm{t}}$ is reached for a finite value of the surface concentration $\delta c=\left[\left|t-\alpha^{2}\right| / \lambda_{4}\right]^{1 / 2}$ and the polymer chain adsorbs. At the transition, $t \rightarrow t_{\mathrm{c}}$, the thickness of the adsorbed layer diverges as

$$
D^{*} \sim \frac{1}{\alpha}\left[\left|t-t_{c}\right| / \lambda_{4}\right]^{-1 / 2}
$$

A finite chain is, of course, effectively adsorbed only if the thickness $D^{*}$ is smaller than its gyration radius. 
3.2 ThE NON-NEUTRAL SURFACE. - When the average concentration of the monolayer does not correspond to the neutral surface condition, $c_{0} \neq c_{\mathrm{s}}^{*}$, the average interaction $\gamma=\gamma_{0}+\alpha c_{0}$ between the polymer and the surface must be added to equation (3.1), similar to equation (2.1)

$$
F_{\mathrm{ps}}=-\frac{\alpha \delta c+\gamma}{D}
$$

The free energy varies now with the surfactant concentration fluctuation $\delta c$ as

$$
F_{\mathrm{t}}=\frac{1}{2} \delta c^{2}\left(t-\alpha^{2}\right)+\frac{1}{4} \lambda_{4} \delta c^{4}-\frac{1}{2} \gamma^{2}-\gamma \alpha \delta c .
$$

For positive values of $\gamma$ (and $\alpha$ ), the average surface tends to adsorb the polymer chain and this effect is enhanced by the fluctuations: the local surfactant concentration $\delta c$ is positive and the thickness of the layer is smaller than on an equivalent homogeneous surface.

For negative values of $\gamma$ (but with $\alpha>0$ ), the equivalent homogeneous surface tends to repel the polymer. When $t$ is decreased, there is a first order transition between depletion and adsorption at a value $t_{0}$. We distinguish between three limits:

$$
\begin{array}{ll}
t_{0}=\alpha^{2}-3 \lambda_{4}\left(-2 \gamma \alpha \lambda_{4}\right)^{2 / 3} / 2 & 0>\gamma \gg-\alpha^{2} / \lambda_{4}^{1 / 2} \\
t_{0}=-2 \gamma \lambda_{4}^{1 / 2}-3 \alpha^{2} / 4 & -3 \alpha^{2} / 8 \lambda_{4}^{1 / 2}>\gamma>-\alpha^{2} / 2 \lambda_{4}^{1 / 2} \\
t_{0}=-\gamma^{2} \lambda_{4} / \alpha^{2} & -\alpha^{2} / 2 \lambda_{4}^{1 / 2} \gg \gamma .
\end{array}
$$

Note that when $t$ is negative, the pure surfactant monolayer undergoes a phase separation and the reference monolayer state used to derive equation (3.8) is that of the denser coexisting phase. As was mentioned above, it is possible to include excluded volume interactions for the polymer chain. However, since the qualitative picture does not change by including these interactions, we do not treat this effect in the present paper.

The adsorption of a single polymer chain on a quenched disordered surface has been studied by several authors [6-10]. If the surface is infinite and the chain is large but finite, there is always a region on the infinite surface with a sufficiently large attractive fluctuation for the chain to adsorb. Thus, the polymer chain always adsorbs on a surface with quenched heterogeneities, but this is very specific to adsorption of a single finite chain and will not be further discussed.

\section{Polymer adsorption on surfactant monolayers: semi-dilute polymer solutions.}

In this section we discuss the adsorption of polymers from a semi-dilute bulk solution on soluble (Gibbs) and insoluble (Langmuir) monolayers. The equilibrium and interfacial properties of the combined polymer-surfactant system can be obtained from the total free energy of the mixed system: $F_{\mathrm{t}}=F_{\mathrm{s}}+F_{\mathrm{p}}+F_{\mathrm{ps}}$. Whereas $F_{\mathrm{s}}$ and $F_{\mathrm{ps}}$ have been discussed in sections 2 and 3 , the interfacial free energy $F_{\mathrm{p}}$ for a semi-dilute polymer solution has to be specified. Within a mean field approach $[3,15]$ that ignores excluded volume correlations, it can be written (in units of $k_{\mathrm{B}} T$ and per unit surface) as a functional of the polymer order parameter $\psi(z)$ related to the local monomer concentration $c_{\mathrm{p}}(z)$ by $\psi^{2}(z)=c_{\mathrm{p}}(z)$

$$
F_{\mathrm{p}}=\int\left(\frac{a^{2}}{6}(\nabla \psi)^{2}+\frac{1}{2} v\left(\psi^{2}-\psi_{\mathrm{b}}^{2}\right)^{2}\right) \mathrm{d} z
$$

Equation (4.1) describes a polymer profile in contact with a polymer reservoir at infinity of concentration $c_{\mathrm{b}}=\psi_{\mathrm{b}}^{2}=\psi^{2}(z \rightarrow \infty)$. The profile obtained from equation (4.1) decays towards 
the imposed bulk value $\psi_{\mathrm{b}}$ at infinity. The gradient term accounts for the elasticity of the polymer chains. The second term describes the excluded volume interaction with a positive excluded volume parameter $v$ in good solvent conditions.

The coupling term $F_{\mathrm{ps}}$ includes only the direct interaction between the surfactant with concentration $c_{\mathrm{s}}=c_{0}+\delta c$ and the monomers at the surface with concentration $\psi_{\mathrm{s}}^{2}=\psi^{2}(z=0)$

$$
F_{\mathrm{ps}}=-a^{2}(\alpha \delta c+\gamma) \psi_{\mathrm{s}}^{2} .
$$

It is convenient to introduce dimensionless concentrations and lengths:

$$
\begin{gathered}
u \equiv z / \xi=z /\left(a^{2} / 3 v \psi_{\mathrm{b}}^{2}\right)^{1 / 2} \\
\phi \equiv \psi / \psi_{\mathrm{b}} .
\end{gathered}
$$

By properly rescaling $F_{\mathrm{t}}, \alpha, t, \lambda_{4}$ and $\gamma: \tilde{F}=F_{\mathrm{t}} /\left(a^{2} \xi \psi_{\mathrm{b}}^{2} / 3 k_{\mathrm{B}} T\right), \tilde{\alpha} \equiv 6 \alpha \xi, \tilde{t} \equiv 3 t \xi a^{-2} \psi_{\mathrm{b}}^{-2}$, $\tilde{\lambda}_{4} \equiv 3 \lambda_{4} \xi a^{-2} \psi_{\mathrm{b}}^{-2}$, and $\tilde{\gamma} \equiv 6 \gamma \xi$ one obtains:

$$
\tilde{F}=\int\left(\frac{1}{2}(\nabla \phi)^{2}+\frac{1}{2}\left(\phi^{2}-1\right)^{2}\right) \mathrm{d} u-\frac{1}{2} \tilde{\alpha} \delta c \phi_{\mathrm{s}}^{2}+\frac{1}{2} \tilde{t} \delta c^{2}+\frac{1}{4} \tilde{\lambda}_{4} \delta c^{4}-\frac{1}{2} \tilde{\gamma} \phi_{\mathrm{s}}^{2} .
$$

The free energy $\tilde{F}$ is a functional of the polymer profile $\{\phi(u)\}$, the polymer surface value $\phi_{\mathrm{s}}=\phi(u=0)$, and the surfactant concentration $\delta c$. Minimizing the free energy, equation (4.4) with respect to the profile $\{\phi(u)\}$ yields the usual order parameter profile for the adsorption of a semi-dilute polymer solution on an ideal solid surface at $z=u=0, \phi(u)=\operatorname{coth}(u+\tilde{b})$, where $\tilde{b}$ is an integration constant related to the surface value of the order parameter by $\phi_{\mathrm{s}}=\operatorname{coth} \tilde{b}$

We then minimize the free energy with respect to $\phi_{\mathrm{s}}$ and obtain a relation between the polymer surface value $\phi_{\mathrm{s}}$ and the surfactant concentration $\delta c$

$$
\delta c+\frac{\tilde{\gamma}}{\tilde{\alpha}}=\frac{\phi_{\mathrm{s}}^{2}-1}{\tilde{\alpha} \phi_{\mathrm{s}}}
$$

We can thus express the total free energy $\tilde{F}$ as a function of the surfactant concentration

$$
\tilde{F}=-\frac{1}{6}\left(\phi_{\mathrm{s}}-1\right)\left(\phi_{\mathrm{s}}^{2}+\phi_{\mathrm{s}}+4\right)+\frac{1}{2} \tilde{t} \delta c^{2}+\frac{1}{4} \tilde{\lambda}_{4} \delta c^{4}
$$

where $\phi_{\mathrm{s}}$ is an implicit function of the surfactant concentration $\delta c$ given by equation (4.5).

At thermodynamic equilibrium, the value of the surfactant concentration is obtained from this free energy and completely determines the polymer adsorption. This is now discussed separately for insoluble and soluble monolayers.

4.1 Adsorption on an insoluble Langmuir monolayer. - When the monolayer is insoluble, the total number of surfactant molecules in the monolayer remains constant and the surfactant concentration is a conserved order parameter. The stability of the monolayer is obtained by studying the convexity of the free energy per unit area $\tilde{F}$; the monolayer becomes unstable if the second derivative of the free energy becomes negative. This defines a spinodal line which separates metastable and unstable regions

$$
\frac{\partial^{2} \tilde{F}}{\partial \delta c^{2}}=\tilde{t}+3 \tilde{\lambda}_{4} \delta c^{2}-\tilde{\alpha}^{2} \frac{\phi_{s}^{3}}{\phi_{s}^{2}+1}=0 .
$$

When $\delta c=0$, the monolayer becomes unstable at a positive value $\tilde{t}=\tilde{t}_{\mathrm{s}}$

$$
\tilde{t}_{\mathrm{s}}=\tilde{\alpha}^{2} \frac{\phi_{\mathrm{s}}^{3}}{1+\phi_{\mathrm{s}}^{2}}
$$


where the polymer order parameter on the surface $\phi_{\mathbf{s}}$ is

$$
\phi_{\mathrm{s}}=\frac{1}{2}\left(\tilde{\gamma}+\sqrt{\tilde{\gamma}^{2}+4}\right)
$$

When $\tilde{t}$ is decreased by changing the temperature, the monolayer undergoes a first order phase transition. The precise location of this transition ( $\tilde{t}_{0}$ as a function of $\left.\delta c\right)$ is obtained by the standard common tangent construction of the free energy $\tilde{F}$. For a fixed value of $\delta c$, the transition occurs at a value $\tilde{t}_{0}$ higher than the spinodal value given by equation (4.7). If $\tilde{t}$ is larger than $\tilde{t}_{0}$, the monolayer remains homogeneous and the polymer does not adsorb. If $\tilde{t}$ is smaller than $\tilde{t}_{0}$, the monolayer phase separates into two phases: a dense surfactant phase onto which the polymer adsorbs and a dilute surfactant phase from which the polymer is depleted.

In the strong adsorption limit, $\tilde{\alpha}^{4}>>\tilde{\lambda}_{4}$, the interaction energy of a monomer with a surfactant molecule is of order $k_{\mathrm{B}} T$, and a first order transition takes place at

$$
\tilde{t}_{0}=\tilde{\alpha}^{6} / 18 \tilde{\lambda}_{4}
$$

At the transition, $t_{0}$ is positive and independent of the polymer concentration in the bulk. The surfactant concentration in the dense phase at the transition is $\delta c=\tilde{\alpha}^{3} / 3 \tilde{\lambda}_{4}$ which is also independent of the polymer bulk concentration.

In the dense surfactant phase, the polymer adsorption is enhanced and the surface order parameter is

$$
\phi_{\mathrm{s}}=\tilde{\gamma}+\tilde{\alpha}^{4} / 3 \tilde{\lambda}_{4}
$$

The relevant experimental quantity is the polymer surface excess $\Gamma=\int_{0}^{\infty}\left(c_{\mathrm{p}}(z)-c_{\mathrm{b}}\right) \mathrm{d} z=$ $c_{\mathrm{b}} \xi\left(\phi_{\mathrm{s}}-1\right)$. In the surfactant dense phase it is independent of the polymer bulk concentration and it is of the order of $\Gamma=a^{-2}$ corresponding to a very strong adsorption.

Although we have discussed here possible phase transitions in Langmuir monolayers induced by polymer adsorption, we have not constructed the global surface phase diagram of the polymer-surfactant mixture. Some general considerations can be found in reference [17]. In principle, the phase diagrams can be calculated using the full free energy of the surfactant monolayer valid in the whole range of concentrations and not only a Landau expansion. The phase diagram of the monolayer contains two transition lines, the "special transition" line between adsorption and depletion for the polymer and a binodal line representing the phase transitions of the surfactant. The "special transition" line only depends on the nominal surfactant concentration $c_{0}$ of the monolayer and has been approximated here by a vertical line in the temperature-surfactant concentration plane. The surfactant binodal line is shifted by the coupling to the adsorption of the polymer. The spinodal line of this transition is given by equation (4.7) with $\delta c=0$ where $t$ is the inverse osmotic compressibility of the monolayer calculated from the second derivative of the surfactant free energy $F_{\mathrm{s}}$. Using the Bragg-Williams form of the free energy, we find that the spinodal line is always shifted to higher temperature by the adsorption of polymer and that the critical concentration corresponding to the maximum of the spinodal line has a higher value than for the pure surfactant. In general, the two lines of the phase diagram meet at a surfactant concentration which can be either smaller or larger than the critical concentration of the surfactant phase transition.

4.2 Adsorption on a soluble Gibbs monolayer. - In a soluble Gibbs monolayer, the surfactant concentration is a non-conserved order parameter and its value is obtained by minimizing the surface free energy, equation (4.4). In the case where the monolayer is strongly attractive for the polymer, i.e., when $\gamma$ is large, the surface free energy has a single minimum 
and the adsorption is enhanced by the coupling between polymer and surfactant. As $\gamma$ is decreased below a critical value $\tilde{\gamma}_{c}=\tilde{\alpha}^{4} /\left(4 \cdot 3^{3 / 2} \tilde{\lambda}_{4}\right)$, (independent of the bulk concentration), the free energy has two minima. As a function of $t$ there can be an exchange of stability between these two minima when $t$ is decreased. At high values of $t$ the change in surfactant concentration is small and the adsorption is weak. For a critical value $t_{0}$, there is a discontinuous first-order transition in the monolayer towards a higher concentration and a much stronger adsorption. This is also the preferred state for $t<t_{0}$.

When the average adsorption vanishes, at large values of $t$, the concentration in the monolayer is $\delta c=\tilde{\alpha} /(2 \tilde{t})=\alpha a^{2} c_{b} / t$ and the polymer surface excess is $\Gamma=\alpha^{2} a^{4} c_{b} /(v t)$ similar to the result obtained in reference [12]. The first order transition occurs at a value $t_{0}$ given by equation (4.10) and at lower values of $t$, the adsorption is strong and the surface excess is of the order of $\Gamma=a^{-2}$

Note that in the soluble case we still consider only direct interactions between polymers and surfactants on the surface. We do not take into account possible polymer-surfactant interaction in the solution and the influence of the surfactant cmc. Another interesting situation where such a condition is satisfied is an interface separating an aqueous solution of surfactant from a polymer solution in an organic solvent. Here there is virtually no interaction of the polymer and surfactant but on the interface.

\section{Polymer adsorption on quenched leterogeneous surfaces.}

We now turn to the case of quenched heterogeneities of the adsorbing surface. A typical example would be heterogeneities due to chemical contaminations which are terminally attached to a solid surface. If the amplitude of the heterogeneity is small, it can be expressed as a sum of Fourier modes. We first discuss the adsorption of a semi-dilute polymer solution onto a solid surface with one single $q$-mode periodic heterogeneity. Two cases are considered: (i) a strongly adsorbing surface with a small periodic modulation; and (ii) a neutral surface whose average is just on the transition line ("special transition") between adsorption and desorption. In the latter case we extend our results to a true random heterogeneity which is a sum of all $q$-modes.

5.1 Strong adsorption on a periodic heterogeneous surface. - The free energy of the polymer solution in contact with a heterogeneous surface is given in the mean-field approximation by the functional $F=F_{\mathrm{p}}+F_{\mathrm{ps}}$.

$$
F / k_{\mathrm{B}} T=\int\left[\frac{a^{2}}{6}(\nabla \psi)^{2}+\frac{1}{2} v\left(\psi^{2}-\psi_{\mathrm{b}}^{2}\right)^{2}\right] \mathrm{d} \mathbf{r}-a^{2} \int \gamma(x) \psi^{2}(\mathbf{r}) \delta(z) \mathrm{d} \mathbf{r}
$$

The first term, $F_{\mathrm{p}}$, is the bulk free energy of the polymer already discussed above. It must be noted, however, that since the surface is heterogeneous, the polymer concentration $c_{\mathrm{p}}=\psi^{2}$ and the gradient term $(\nabla \psi)^{2}$ vary both in the $x$-direction (parallel to the surface) and the $z$-direction (perpendicular to the surface). The second term, $F_{\mathrm{ps}}$, is the direct surface term: $\gamma$ describes the (assumed attractive) interaction between the polymer and the surface and is coupled to the polymer concentration on the surface, $\psi_{s}^{2}$.

As our example of a periodic heterogeneous surface, we choose a coupling constant $\gamma(x)$ that varies periodically along the $x$-direction with a wavevector $q$.

$$
\gamma(x)=\gamma_{1}+\delta \gamma \cos q x .
$$

In the strong adsorption limit $1 / \gamma_{1}$ is of order of the monomer size $a$. We consider here only weak heterogeneities for which $\delta \gamma \ll \gamma_{1}$. As was done above, all lengths are rescaled by the 
bulk correlation length $\xi=a / \sqrt{3 v c_{\mathrm{b}}}: u \equiv z / \xi, w \equiv x / \xi$ and $k \equiv q \xi$, and the dimensionless order parameter is $\phi \equiv \psi / \psi_{\mathrm{b}}$ with a surface value $\phi(0)=\phi_{\mathrm{s}}$.

As the surface is only heterogeneous but otherwise flat and smooth, the equations obtained by minimizing the free energy functional are the same as on an ideal surface

$$
\begin{aligned}
& \nabla^{2} \phi-2 \phi\left(\phi^{2}-1\right)=0 \\
& \left.\frac{\partial \phi}{\partial u}\right|_{u=0}+\tilde{\gamma}(w) \phi_{\mathrm{s}}=0
\end{aligned}
$$

where the dimensionless interaction parameter is $\tilde{\gamma}=\tilde{\gamma}_{1}+\delta \tilde{\gamma} \cos k w, \tilde{\gamma} \equiv 6 \gamma \xi, \tilde{\gamma}_{1} \equiv 6 \gamma_{1} \xi$, and $\delta \tilde{\gamma} \equiv 6 \delta \gamma \xi$.

In the weak heterogeneity limit the polymer concentration profile can be found perturbatively as an expansion in $\delta \tilde{\gamma}$. At zeroth order in the heterogeneity strength, the concentration profile is exactly the same as for an ideal homogeneous surface, $\phi_{0}(u)=\operatorname{coth}(u+\tilde{b})$, where the dimensionless extrapolation length $\tilde{b}=b / \xi$ is such that $\sinh 2 \tilde{b}=2 / \tilde{\gamma}_{1}$.

Since the first order perturbation to this profile is periodic with a wavevector $q$, the order parameter can be written as

$$
\phi=\phi_{0}+\delta \tilde{\gamma} \phi_{1}(u) \cos k w .
$$

Substitution in equation (5.3) gives the following equation for $\phi_{1}$ :

$$
\begin{aligned}
\kappa_{1}^{2} \phi_{1}-\frac{\partial^{2} \phi_{1}}{\partial u^{2}}+\frac{6 \phi_{1}}{\sinh ^{2}(u+\tilde{b})} & =0 \\
\frac{\partial \phi_{1}}{\partial u}+\tilde{\gamma} \phi_{1}(0)+\phi_{0}(0) & =0
\end{aligned}
$$

where $\kappa_{1}^{2} \equiv 4+k^{2}$.

Far away from the solid surface, the order parameter is $\phi=1$ and $\phi_{1}$ must vanish. The correction to the order parameter profile is then given by

$$
\phi_{1}(u)=A \mathrm{e}^{-\kappa_{1}(u+\tilde{b})}\left(\operatorname{coth}^{2}(u+\tilde{b})+\kappa_{1} \operatorname{coth}(u+\tilde{b})+\frac{\kappa_{1}^{2}-1}{3}\right)
$$

where $A$ is an integration constant. This correction decreases exponentially with the dimensionless decay length $\kappa_{1}^{-1}$ at distances far from the solid surface. At small wavevectors $(q \xi \ll 1)$, this decay length is of the order of the bulk correlation length $\xi$, whereas at large wavevectors $(q \xi \gg 1)$ the decay length is of the order of the period of the heterogeneity $q^{-1}$

The integration constant $A$ can be estimated in the two limits: $\kappa_{1} \tilde{b} \gg 1$ and $\kappa_{1} \tilde{b} \ll 1$

$$
\begin{array}{ll}
A=\tilde{b}^{2} & \kappa_{1} \tilde{b} \ll 1 \\
A=\frac{3}{\tilde{b} \kappa_{1}^{3}} \mathrm{e}^{\kappa_{1} \tilde{b}} & \kappa_{1} \tilde{b} \gg 1 .
\end{array}
$$

The quantity of experimental relevance is the surface excess $\Gamma$. We define here a dimensionless surface excess $\tilde{\Gamma} \equiv \Gamma / c_{b} \xi$. For a homogeneous surface $\tilde{\Gamma}_{0}=\tilde{\gamma}$. In the presence of a periodic heterogeneity

$$
\tilde{\Gamma}=\tilde{\Gamma}_{0}+2 \delta \tilde{\gamma} \cos k w \int_{0}^{+\infty} \phi_{0} \phi_{1} \mathrm{~d} u .
$$

In the limit of small wavevector $q b \ll 1,\left(\kappa_{1} \tilde{b} \ll 1\right)$

$$
\tilde{\Gamma}=\tilde{\Gamma}_{0}+\delta \tilde{\gamma} \cos k w \text {. }
$$


The relative change in the surface excess is $\delta \Gamma / \Gamma_{0}=\left(\delta \gamma / \gamma_{1}\right) \cos q x$. As soon as the wavelength of the heterogeneity is larger than the extrapolation length $b, q b>1$, the fluctuation of the surface excess follows the heterogeneity of the solid surface. In the strong adsorption limit considered here, the extrapolation length $b$ is of the order of the monomer size $a$. In practice, all heterogeneities of the solid surface will thus create fluctuations of the surface excess.

Note that the response of an adsorbed polymer layer to a heterogeneity is different from the response of a thin wetting film for which it has been shown $[21,22]$ that fluctuations with a wavelength smaller than a finite healing length (in general larger than the film thickness) are damped. For the liquid film, the damping of the surface corrugations is due to surface tension between the film and the vapor. In the polymer adsorption problem, there is no interfacial tension between the adsorbed layer and the bulk solution. This result can easily be understood if one looks at the local structure of an adsorbed layer. Locally, the adsorbed layer has the structure of a semi-dilute polymer solution, it thus responds to perturbations with a wavelength larger than the local correlation length. The heterogeneity produces a perturbation on the surface where the local correlation length is the extrapolation length $b$. Hence, it perturbs the concentration profile as soon as the wavelength is larger than $b$.

Although this result has been obtained within the mean-field approximation, the same physical picture prevails when excluded volume interactions are taken into account. This has been checked quantitatively using the Cahn - de Gennes free energy appropriate for polymers in a good solvent.

In the limit of very small wavelength heterogeneities $(q b \gg 1)$, the fluctuation in the surface excess is small and in the mean field approximation its decay length is the bulk correlation length $\xi$

$$
\frac{\delta \Gamma}{\Gamma_{0}}=\frac{\delta \gamma}{\gamma_{1}} \frac{\xi^{2}}{b^{2}} \frac{1}{4+q^{2} \xi^{2}}
$$

5.2 Neutral Periodic and disordered surfaces. - A neutral surface with chemical heterogeneities is a surface whose averaged behavior has no preference to the polymer adsorption. In the absence of heterogeneity, the concentration profile is flat, the reduced order parameter is $\phi=1$ and the surface excess vanishes $\Gamma_{0}=0$. The coupling constant between the polymer and the surface is given by equation (5.2) with $\gamma_{1}=\tilde{\gamma}_{1}=0$. We consider here only the limit where the amplitude $\delta \gamma$ of the heterogeneity is small.

The order parameter profile is also obtained from equation (5.1). In order to find this profile, we expand $\phi$ in Fourier series

$$
\phi=1+\sum_{n} \phi_{n}(u) \cos n k w .
$$

We then expand the polymer profile in powers of the reduced heterogeneity $\delta \tilde{\gamma}$. Up to first order in $\delta \tilde{\gamma}$ only the first harmonic $\phi_{1}$ does not vanish. It satisfies the equation

$$
\begin{array}{r}
\left(\kappa_{1}^{2}-\frac{\partial^{2}}{\partial u^{2}}\right) \phi_{1}=0 \\
\left.\frac{\partial \phi_{1}}{\partial u}\right|_{0}+\delta \tilde{\gamma} \cos k w=0
\end{array}
$$

where $k_{1}^{2} \equiv 4+k^{2}$ as in the previous section.

The first order correction to the polymer profile is thus

$$
\phi_{1}(u)=\frac{\delta \tilde{\gamma}}{\kappa_{1}} \exp \left(-\kappa_{1} u\right) .
$$


To first order in $\delta \tilde{\gamma}$, the surface excess still vanishes and one must thus expand up to second order in $\delta \tilde{\gamma}$ in order to obtain the surface excess. For symmetry reasons only the first two Fourier components $\phi_{0}$ and $\phi_{2}$ have contributions to order $(\delta \tilde{\gamma})^{2}$. The calculation of the surface excess requires only the contribution of $\phi_{0}$ to second order in $\delta \tilde{\gamma}, \phi_{0}^{(2)}$ It is given by

$$
\begin{aligned}
4 \phi_{0}^{(2)}-\frac{\partial^{2} \phi_{0}^{(2)}}{\partial^{2} u} & =-\frac{3 \delta \tilde{\gamma}^{2}}{\kappa_{1}^{2}} \exp \left(-2 \kappa_{1} u\right) \\
\frac{\partial \phi_{0}^{(2)}}{\partial u} & =-\frac{\delta \tilde{\gamma}^{2}}{2 \kappa_{1}}
\end{aligned}
$$

This leads to

$$
\phi_{0}^{(2)}=\frac{\delta \tilde{\gamma}^{2}}{4 \kappa_{1}} \frac{k^{2}}{3+k^{2}} \mathrm{e}^{-2 u}+\frac{3 \delta \tilde{\gamma}^{2}}{4\left(3+k^{2}\right)+\kappa_{1}^{2}} \mathrm{e}^{-2 \kappa_{1} u}
$$

Therefore, we can determine the reduced surface excess $\tilde{\Gamma}=\int_{0}^{+\infty} \mathrm{d} u\left[\frac{1}{2} \phi_{1}^{2}+2 \phi_{0}^{(2)}\right]$

$$
\tilde{\Gamma}=\frac{\delta \tilde{\gamma}^{2}}{4 r_{1}^{3}}\left(2+k^{2}\right)
$$

Thus, although the average surface is neutral and $\Gamma_{0}=0$, on the heterogeneous surface $\Gamma>0$ and the heterogeneity enhances adsorption, as in the annealed case discussed in section 3 .

If the wavevector of heterogeneity is small, $q \xi \ll 1, \Gamma$ decreases with the bulk concentration

$$
\Gamma=\frac{3}{4} \frac{(\delta \gamma)^{2} a^{2}}{v} \xi \sim \frac{1}{\sqrt{c_{\mathrm{b}}}} .
$$

If $q \xi \gg 1$, the effect of the heterogeneity is damped and $\Gamma$ is a decreasing function of $q$ :

$$
\Gamma=\frac{3(\delta \gamma)^{2} a^{2}}{v q}
$$

The surface excess becomes thus very small when the wavevector is larger than the bulk correlation length of the solution. If the average surface is neutral, the concentration profile is roughly independent of $x$ and the local correlation length on the surface is equal to $\xi$, and the adsorption layer follows heterogeneities with a wavelength larger than the bulk correlation length $\xi$.

If the heterogeneity of the surface is not periodic but random, we characterize it by its power spectrum $G(\mathbf{q})=\int \mathrm{d} \rho\langle\delta \gamma(0) \delta \gamma(\rho)\rangle e^{i q_{1} \rho}$ which for simplicity is taken to be Lorentzian

$$
G(\mathbf{q})=\frac{\Delta^{2}}{q^{2}+\xi_{\mathrm{s}}^{-2}}
$$

The correlation length $\xi_{\mathrm{s}}$ of the surface heterogeneity is supposed to be much smaller than the bulk correlation length $\xi, \xi_{\text {s }} \ll \xi$.

The surface excess can be obtained in a first approximation by summing over the contributions of the various wavevectors $q$. For each wave vector the contribution to the surface excess $\Gamma$ is given by equation (5.16).

$$
\Gamma=\int \frac{\mathrm{dq}}{4 \pi^{2}} \frac{3 \xi a^{2}}{v} \frac{2+k^{2}}{\kappa_{1}^{3}} G(\mathbf{q}) .
$$


In the limit where $\xi_{\mathrm{s}} \ll \xi$ we find

$$
\Gamma=\frac{3}{4} \frac{\Delta^{2} \xi_{\mathrm{s}} a^{2}}{v}
$$

A naive mean field theory predicts thus for random heterogeneities a positive surface excess corresponding to an effective adsorption and independent of the bulk concentration. Although this result has been obtained here through an expansion for small heterogeneity, we believe that it is more general and that on any surface neutral on average to the polymer, the presence of heterogeneities induces an effective adsorption and leads to a positive surface excess $\Gamma$.

In the limit where the heterogeneity is very strong, this can be justified by the following qualitative argument. The heterogeneous surface can be viewed as a checkerboard where a certain fraction of the sites are strongly attractive, (a monomer gaining a fluctuation energy $\Delta$ upon adsorption) and the rest are strongly repulsive, (the adsorption of a monomer costing a fluctuation energy $-\Delta$ ). The size of the sites is of the order of the surface correlation length of the heterogeneity $\xi_{\mathrm{s}}$, and on a neutral surface half of the sites are repulsive and half are attractive. If the energy gained by a monomer upon adsorption is larger than $k_{\mathrm{B}} T$ entropic effects play little role and all the adsorbing sites are covered by a monomer. The repulsive sites of the surface can be avoided by the polymer chains by making small loops towards the bulk. Each loop costs an energy $k_{\mathrm{B}} T$ much smaller than the adsorption energy. At equilibrium, we thus expect that half of the sites on the surface are covered. This leads to a large positive surface excess $\Gamma \simeq a^{-2}$.

Finally, one should notice that all the discussion on quenched heterogeneity has been done at the mean field level. For a semi-dilute polymer solution in a good solvent, one must take into account the excluded volume correlations. The concentration profile of an adsorbed solution is then singular in the vicinity of a surface (proximal effect). It is not clear however how relevant the quenched disorder is for this proximal effect and the precise concentration profile in the vicinity of a heterogeneous surface is not known. One can, nevertheless, speculate that the qualitative trend given by the mean field calculation is correct and that the heterogeneity enhances adsorption. In Appendix B we present a second-order cumulant expansion for the poymer surface excess for both quenched and annealed disorder. On a mean filed level, we can show from general considerations that the disorder enhances polymer adsorption.

\section{Concluding remarks.}

The main finding of this paper is that polymer adsorption is enhanced by surface heterogeneity. In all studied cases, for annealed and quenched heterogeneities, the fluctuations due to the non-homogeneous surface cause local fluctuations in the polymer surface excess and increase its overall mean. We also have shown that at least for small heterogeneities, the increase in the average surface excess is larger for annealed than for quenched disorder (Appendix B).

Our findings on the annealed heterogeneity are of relevance to experiments on the adsorption of polymer solutions on interfaces supporting a surfactant monolayer $[18,19]$. With a Langmuir (insoluble) monolayer and a hydrosoluble polymer in the aqueous subphase, pressure-area isotherms can be measured by applying a lateral surface pressure. A slightly more complex situation is to use a Langmuir monolayer composed of a mixture of two insoluble surfactants or amphiphiles such as DPPC and cholesterol [23, 24].

For soluble surfactants, one can look at changes of the surface tension as function of the surfactant and polymer concentrations. The polymer and the surfactant interact then not only at the interface but also in the bulk. The influence of polymers on the surfactant micelles and other mesophases can be of importance as was discussed by Cabane et al. [16]. It then affects the chemical potential of the surfactant in the bulk solution and thus the properties of 
the monolayer that is in equilibrium with this bulk solution. Although experiments are easier to carry out at the water/air interface, the interpretation could be simpler for an oil/water interface where the polymer and surfactant overlap only at the interface.

The most relevant and measurable polymer quantity is the surface excess $\Gamma$, which measures the integrated affinity of the polymer to the surface. The entire polymer profile $c_{\mathrm{p}}(z)$ and its surface value $c_{\mathrm{p}}(0)$ can also be measured, for example by neutron scattering [4]. Any correlation found between the presence of surfactant and polymer will elucidate on their interaction. In the present work we have described several such correlations in some simple situations.

Possible extensions of our work will be to include an interaction between the polymer and surfactant in the bulk, in addition to their presence on the surface. The surfactant may influence the polymer profile and the extent of its adsorption, whereas the polymer can have complex interactions with the surfactant micelles: shift of the cmc, concatenation of micelles by single polymer chains, transitions between globular and cylindrical micelles, transitions between micellar and other phases such as lamellae, vesicles, cubic phases for concentrated surfactant solutions. Finally, we note that polymer adsorption on surfaces with quenched heterogeneities is also of great interest because it is virtually impossible to produce a perfectly homogeneous surface. They probably will be studied in more details in the future.

\section{Acknowledgements.}

One of us (DA) would like to thank Institut C. Sadron for its hospitality. He also acknowledges partial support from the Israel Academy of Sciences and Humanities.

\section{Appendix A.}

Single chain on off-critical monolayers.

When the reference concentration $c_{0}$ is not the critical concentration for the surfactant phase separation $c_{\mathrm{k}}$, the Landau expansion of the surfactant free energy is given by equation (2.4) with a non vanishing cubic term in $\delta c$. The coefficient $\lambda_{3}$ of this cubic term is positive if $c_{0}>c_{k}$ and negative if $c_{0}<c_{\mathrm{k}}$. Here, we discuss single chain adsorption on such a monolayer in the neutral case where $\gamma=\gamma_{0}+\alpha c_{0}=0$. The thickness of the adsorbed polymer chain $D$ is a function of the local concentration in the monolayer. It is independent of the coefficient $\lambda_{3}$ and is given by the values found at the critical point. The total free energy as a function of the surfactant concentration is obtained along the same lines as equation (3.4),

$$
F_{\mathrm{t}}=\frac{1}{2} \delta c^{2}\left(t-\alpha^{2}\right)+\frac{1}{3} \lambda_{3} \delta c^{3}+\frac{1}{4} \lambda_{4} \delta c^{4}
$$

If $\lambda_{3}$ is positive, (for surfactant concentrations larger than the critical value), the polymer chain adsorbs when $t<t_{\mathrm{c}}=a^{2}$ and the change in the monomer concentration due to the adsorption is continuous: $\delta c=\left|t-t_{\mathrm{c}}\right| / \lambda_{3}$. The thickness of the adsorbed layer diverges at the transition as

$$
D^{*} \sim \frac{1}{\alpha}\left[\left|t-t_{\mathrm{c}}\right| / \lambda_{3}\right]^{-1}
$$

with a different power law divergence than when $\lambda_{3}=0$, equation (3.5).

If $\lambda_{3}$ is negative, (for surfactant concentrations smaller than the critical value), the transition corresponding to the adsorption of the polymer chain is discontinuous and occurs at $t_{0}=$ 
$\alpha^{2}+2 \lambda_{3}^{2} /\left(9 \lambda_{4}\right)$. The jump in the surfactant concentration at the place where the chain adsorbs is $\delta c=-2 \lambda_{3} /\left(3 \lambda_{4}\right)$. The thickness of the adsorbed layer is then

$$
D^{*} \sim-\frac{3 \lambda_{4}}{2 \alpha \lambda_{3}}
$$

it remains finite at the transition as long as $\lambda_{3}<0$.

\section{Appendix B.}

Cumulant expansion for quenched and annealed surface excess.

Comparison between quenched and annealed surface disorder is possible in a simple case where the surface impurities $\{h(\mathrm{x})\}$ are assumed to have an uncorrelated Gaussian distribution about a zero mean

$$
\begin{aligned}
& \left\langle h(\mathbf{x}) h\left(\mathbf{x}^{\prime}\right)\right\rangle=\Delta^{2} \delta\left(\mathbf{x}-\mathbf{x}^{\prime}\right) \\
& \langle h(\mathbf{x})\rangle=0 .
\end{aligned}
$$

For polymer adsorption, the polymer surface excess defined as

$$
\Gamma=\frac{1}{A} \int\left(\psi^{2}(\mathbf{r})-\psi_{\mathrm{b}}^{2}\right) \mathrm{d} \mathbf{r}
$$

where $A$ is the surface area, is averaged differently for quenched and annealed disorders [13].

Whereas for the annealed case $\Gamma_{\mathrm{a}}$ is

$$
\Gamma_{\mathrm{a}}=\frac{\left\langle\left\langle\Gamma \mathrm{e}^{-\mathcal{H}_{1}}\right\rangle_{0}\right\rangle_{h}}{\left\langle\left\langle\mathrm{e}^{-\mathcal{H}_{1}}\right\rangle_{0}\right\rangle_{h}}
$$

for the quenched case $\Gamma_{q}$ is

$$
\Gamma_{\mathrm{q}}=\left\langle\frac{\left\langle\Gamma \mathrm{e}^{-\mathcal{H}_{1}}\right\rangle_{0}}{\left\langle\mathrm{e}^{-\mathcal{H}_{1}}\right\rangle_{0}}\right\rangle_{h}
$$

where $\langle\mathcal{O}\rangle_{h} \equiv \int \mathcal{D} h P(h) \mathcal{O} / \int \mathcal{D} h P(h)$ is the average taken over the Gaussian surface impuricy distribution $P(h)$, and $\{\mathcal{O}\}_{0}$ is the thermal average taken with the non-random polymer "Hamiltonian" $\mathcal{H}_{0}:\langle\mathcal{O}\rangle_{0} \equiv \int \mathcal{D} \psi \exp \left(-\mathcal{H}_{0}\right) \mathcal{O} / \int \mathcal{D} \psi \exp \left(-\mathcal{H}_{0}\right)$. As in sections 3, the polymer Hamiltonian $\mathcal{H}_{0}$ depends on the order parameter $\psi$ and the polymer concentration $c_{\mathrm{p}}(\mathbf{r})$ is just $\psi^{2}(\mathbf{r})$

The polymer-surface interaction "IIamiltonian" $\mathcal{H}_{1}$ appearing in equations (B3, B4) just represents the bilinear coupling between the monomer surface concentration and the impurity degree of freedom

$$
\mathcal{H}_{1}=\int \mathrm{d} \mathbf{r} h(\mathbf{r}) \psi^{2}(\mathbf{r}) \delta(z) .
$$

Expanding $\Gamma_{\mathrm{a}}$ and $\Gamma_{\mathrm{q}}$ to second order in a cumulant expansion we get

$$
\begin{aligned}
& \Gamma_{\mathrm{a}}=\Gamma_{0}-\frac{1}{2} \Gamma_{0}\left\langle\left\langle\mathcal{H}_{1}^{2}\right\rangle_{0}\right\rangle_{h}+\frac{1}{2}\left\langle\Gamma\left\langle\mathcal{H}_{1}^{2}\right\rangle_{h}\right\rangle_{0} \\
& \Gamma_{\mathrm{q}}=\Gamma_{\mathrm{a}}-\Gamma_{0}\left\langle\left\langle\mathcal{H}_{1}\right\rangle_{0}^{2}\right\rangle_{h}+\left\langle\left\langle\Gamma \mathcal{H}_{1}\right\rangle_{0}\left\langle\mathcal{H}_{1}\right\rangle_{0}\right\rangle_{h}
\end{aligned}
$$


where $\Gamma_{0}=\langle\Gamma\rangle_{0}$ is the surface excess of the pure polymer system, and $\Gamma_{0}=0$ for a neutral surface.

From equation (B6) one sees that even to second order in the cumulant expansion the three surface excesses: $\Gamma_{0}, \Gamma_{a}$ and $\Gamma_{q}$ are not equal. In this paper we show for several cases that annealed and quenched disorder enhance polymer adsorption, $\Gamma_{a}>\Gamma_{0}$ and $\Gamma_{q}>\Gamma_{0}$. We believe that this is a general result and below we outline a proof that $\Gamma_{a}>\Gamma_{q}>\Gamma_{0}$ within second order of the cumulant expansion.

Carrying out the averages over the impurity degrees of freedom $\{h\}$ in equations (B5, B6), for the uncorrelated Gaussian distribution we obtain

$$
\begin{gathered}
\Gamma_{\mathrm{a}}=\Delta^{2} \int \mathrm{d} \mathbf{r}\left\langle\left(\psi^{2}(\mathbf{r})-\psi_{\mathrm{b}}^{2}\right) \psi^{4}(0)\right\rangle_{0} \\
\Gamma_{\mathrm{a}}-\Gamma_{\mathrm{q}}=\Delta^{2} \int \mathrm{d} \mathbf{r}\left\langle\left(\psi^{2}(\mathbf{r})-\psi_{\mathrm{b}}^{2}\right) \psi^{2}(0)\right\rangle_{0}\left\langle\psi^{2}(0)\right\rangle_{0}
\end{gathered}
$$

Since the correlations $(\ldots\rangle_{0}$ in equation (B7) involve a $\psi^{4}$ theory they cannot be calculated exactly. However, using Wick's theorem for $\delta \psi(\mathbf{r})=\psi(\mathbf{r})-\psi_{\mathrm{b}}$ and $\delta \psi(0)=\psi(0)-\psi_{\mathrm{b}}$, it can be shown that $\Gamma_{\mathrm{a}}, \Gamma_{\mathrm{q}}$ and $\Gamma_{\mathrm{a}}$-' are each a sum of positive correlation terms. These correlations have a generic form: $\left\langle\delta \psi^{n}(r) \delta \psi^{m}(0)\right\rangle_{0}$ where $n$ and $m$ are $(1,1),(2,2),(1,3),(2,4),(2,0)$ and $(0,2)$. All these correlation functions are positive and decay to zero for distances $r$ larger than the bulk correlation length $\xi$. This leads to the desired inequalities: $\Gamma_{a}>\Gamma_{q}>\Gamma_{0}$ for the surface excess.

In summary, we are suggesting an enhancement of the polymer surface excess in presence of impurities. Annealed disorder, where the surface can rearrange itself, yields an even higher value of the surface excess than the quenched case. This enhancement is based on the fact that the disorder is assumed to lie only on the surface and that the polymer reaches its bulk concentration within a distance $\xi$ from the surface. Due to the polymer elasticity, the correlation length $\xi$ is independent of the surface disorder. In addition, for semi-dilute polymer solutions, the polymer concentration $c_{\mathrm{p}}(\mathbf{r})$ is not the order parameter $\psi(\mathbf{r})$ but the square of it $[14,15]$. The inequality we found is strongly based on this fact since the disorder acts like a surface random interaction and not as a random field [13] (coupled linearly to the order parameter). One should, however, keep in mind that the results obtained here heavily rely on the mean field approximation which ignores excluded volume correlations and thus does not take fully into account the concentration fluctuations.

\section{References}

[1] Napper D. H., Polymeric Stabilization of Colloidal Dispersions (Academic Press: London, 1983).

[2] Cohen-Stuart M. A., Cosgrove T., Vincent B., Adv. Coll. Int. Sci. 24 (1986) 143.

[3] de Gennes P. G., Adv. Coll. Int. Sci. 27 (1987) 189.

[4] Auvray L., Cotton J. P., Macromolecules 20 (1988) 202.

[ن] Kawaguchi M., Takahashi A., Macromolecules 16 (1983) 1465.

[6] Baumgärtner A., Muthukumar M., J. Chem. Phys. 87 (1987) 3082.

[7] Cates M. E., Ball R., J. Phys. France 49 (1988) 2009.

[8] Edwards S. F., Mutlukumar M., J. Chem. Phys. 89 (1988) 2435.

[9] Hone D., Ji H., Pincus P. A., Macromolecules 20 (1987) 2543; 
Ji H., Hone D., Macromolecules 21 (1988) 2600.

[10] Blunt M., Barford W., Ball R., Macromolecules 22 (1989) 1458.

[11] Odijk T., Macromolecules 23 (1990) 1875.

[12] Andelman D., Joanny J. F., Macromolecules 24 (1991) 6040.

[13] Lubensky T. C., Ill-Condensed Matter, Les Houches 1978, R. Balian, R. Maynard and G. Toulouse Eds. (North-Holland, Amsterdam, 1979).

[14] Edwards S. F., Proc. Phys. Soc. 85 (1965) 613; ibid 88 (1966) 265.

[15] de Gennes P. G., Scaling Concepts in Polymer Physics (Cornell University Press, Ithaca, 1979).

[16] Cabane B., Duplessix R., J. Phys. France 48 (1987) 651.

[17] de Gennes P. G., J. Phys. Chem. 94 (1990) 8407.

[18] Chari K., Hossain T., J. Phys. Chem. 95 (1991) 3302.

[19] Lionti-Addad S., DiMeglio J. M., Langmuir 8 (1992) 324.

[20] Adamson A. W., Physical Chemistry of Surfaces (Wiley Interscience, New York, 1990).

[21] Andelman D., Joanny J.-F., Robbins M. O., Europhys. Lett. 3 (1988) 729.

[22] Robbins M. O., Andelman D., Joanny J.-F., Phys. Rev. A 43 (1991) 4344.

[23] Subramaniam S., McConnell H., J. Phys. Chem. 91 (1987) 1715.

[24] Seul M., Sammon J., Phys. Rev. Lett. 64 (1990) 1903. 